\title{
Ecological and Evolutionary Consequences of Viral Plasticity
}

\author{
Melinda Choua ${ }^{1}$ and Juan A. Bonachela ${ }^{1,2, \star}$
}

1. Marine Population Modeling Group, Department of Mathematics and Statistics, University of Strathclyde, Glasgow G1 1XH, Scotland, United Kingdom; 2. Department of Ecology, Evolution, and Natural Resources, Rutgers University, New Brunswick, New Jersey 08901

Submitted May 4, 2018; Accepted October 2, 2018; Electronically published February 12, 2019

Online enhancements: appendixes.

\begin{abstract}
AвSTRACT: Viruses use the host machinery to replicate, and their performance thus depends on the host's physiological state. For bacteriophages, this link between host and viral performance has been characterized empirically and with intracellular theories. Such theories are too detailed to be included in models that study host-phage interactions in the long term, which hinders our understanding of systems that range from pathogens infecting gut bacteria to marine phage shaping the oceans. Here, we combined data and models to study the short- and long-term consequences that host physiology has on bacteriophage performance. We compiled data showing the dependence of lytic-phage traits on host growth rate (referred to as viral phenotypic plasticity) to deduce simple expressions that represent such plasticity. Including these expressions in a standard host-phage model allowed us to understand mechanistically how viral plasticity affects emergent evolutionary strategies and the population dynamics associated with different environmental scenarios including, for example, nutrient pulses or host starvation. Moreover, we show that plasticity on the offspring number drives the phage ecological and evolutionary dynamics by reinforcing feedbacks between host, virus, and environment. Standard models neglect viral plasticity, which therefore handicaps their predictive ability in realistic scenarios. Our results highlight the importance of viral plasticity to unravel host-phage interactions and the need of laboratory and field experiments to characterize viral plastic responses across systems.
\end{abstract}

Keywords: bacteriophage, host-virus models, eco-evolutionary dynamics.

\section{Introduction}

In the past three decades, advances in virology have unveiled the key role of viruses in a multitude of different ecosystems. From microbiomes to the open ocean, viruses can affect any trophic level, showing a remarkable variety of life strategies that are shaped by viral reproductive mode and life-history traits. Let us focus on viruses that infect bacteria (bacteriophages). The cell lysis induced by marine phages, for example, removes up to $40 \%$ of marine bacteria, and the resulting par-

\footnotetext{
* Corresponding author; email: juan.bonachela@rutgers.edu. ORCIDs: Bonachela, http://orcid.org/0000-0002-3316-8120.

Am. Nat. 2019. Vol. 193, pp. 346-358. (C) 2019 by The University of Chicago. 0003-0147/2019/19303-58448\$15.00. All rights reserved. DOI: $10.1086 / 701668$
}

ticulate and dissolved organic matter thus alter the nutrient paths in the microbial loop (Fuhrman 1999; Abedon et al. 2009; Breitbart 2012). Phages can also affect the bacterial part of the human microbiota, as they can infect biofilms (Abedon 2011), including gastrointestinal bacteria. Lytic phages have been suggested as an alternative to antibiotics (phage therapy; Weld et al. 2004) and, more generally, to treat bacterial infections in plants and animals of commercial interest for the food industry (see Golec et al. 2014 and references therein). Thus, understanding the dynamic interaction between host and phage in the short and long term is essential to making reliable predictions of high biological, medical, and commercial importance.

For obligately lytic phages, the infection cycle starts when the virus encounters and attaches to the host cell (e.g., Calendar and Abedon 2006; see fig. J1; figs. A1-A3, B1, F1, H1, J1J6 are available online). The phage then perforates the cell's membrane and injects its genome into the cytoplasm. The first produced viral components divert the host synthesis machinery, inhibiting host replication. Host DNA is degraded, and host components (nucleotides, ribosomes, ATP, etc.) are used for the replication of the viral genome and synthesis of proteins that will constitute the viral offspring. Late proteins, mostly structural, are assembled to compose the new virions, which are released when the so-called holin gene is expressed to facilitate host membrane lysis. This lytic cycle defines the main viral traits (Weinbauer 2004): (i) adsorption rate, or rate of successful encounters between host and virus; (ii) eclipse period, or time between adsorption and the assembly of the first virion; (iii) maturation rate, or rate of virion assembly; (iv) latent period, or time between adsorption and cell lysis; and (v) burst size, or offspring number. Adsorption depends on multiple factors, from the turbulence of the medium (Berg and Purcell 1977) to phage morphology and host receptors (Schwartz 1976). Although the degree of dependence of the eclipse period on the host differs across phages (e.g., T4 uses host RNA polymerase, whereas T7 also uses its own), host ribosomes are used for viral protein synthesis in all cases (Calendar and Abedon 2006; Walsh and Mohr 2011). Virion assembly and DNA packaging rely on host ATP as energy source (Calendar and Abedon 2006), host 
ribosome number and elongation rate (You et al. 2002), and host metabolic rates (Hadas et al. 1997). On the other hand, although it is not clear what determines the timing of lysis, the timing of the holin gene expression initiating this process is known to depend on both phage and host (Abedon et al. 2001). This timing influences not only viral generation time but also the final burst size (Gnezda-Meijer et al. 2006), a relationship traditionally formulated as a trade-off by which larger offspring numbers require longer latent periods (Wang 2006). Deeply affected by the maturation rate, this number of virions per infection can show a wide range of values and therefore is not the result of mere host nucleotide recycling (You et al. 2002; Brown et al. 2006; Maat et al. 2016).

Phage performance depends, in consequence, on host performance, for example, host population number and physiological state (Wang et al. 1996). The latter has been documented with experiments that follow one single infection cycle (one-step growth) to measure mainly latent period or burst size with a variety of hosts in a diversity of environments (e.g., Webb et al. 1982; Abedon 1989; Kokjohn et al. 1991; Proctor et al. 1993; Middelboe 2000; Abedon et al. 2003; Gnezda-Meijer et al. 2006; Birch et al. 2012; Golec et al. 2014). In all cases, an improvement in the host growth rate correlated with an increase in burst size and/or a shortening of the latent period (depending on the measured trait). A mechanistic understanding of such trends is more elusive, fundamentally due to a limited knowledge about what determines the timing of lysis and the offspring number. Although difficult to find, comprehensive studies that explore simultaneously how all these viral traits are affected by the host physiological state (Hadas et al. 1997; You et al. 2002) enable a more complete picture of the lytic cycle that may provide key pieces to this puzzle.

The experiments above explore what has been referred to as viral phenotypic plasticity (e.g., Abedon et al. 2001), broadly understood as short-term changes in phage trait values triggered by changes in the host, effectively the phage's environment. This plasticity is, however, typically ignored in phage studies. For example, most experiments compiling information about phage traits are conducted ensuring maximal rates for host growth (see discussion in, e.g., Hadas et al. 1997). In nature, however, the situations in which the host grows at its maximum rate are more the exception than the rule. As a consequence, theory or field conclusions built on such trait values are potentially biased, including viral count and effect on the microbial community structure, population density, and dynamics. From a theoretical point of view, intracellular descriptions replicate singleinfection-cycle data (You et al. 2002), but their level of detail renders these models computationally expensive and difficult to parametrize (Birch et al. 2012) and therefore impractical for the study of the long-term behavior of any specific host-virus system. Ecosystem models, for instance, rarely include viruses, or they are included (due to computational constraints) with simplified terms that neglect plasticity (Mateus 2017). On the other hand, optimal (i.e., fitnessmaximizing) latent periods have been estimated using fixed viral traits and/or fixed host concentrations accounting for host quality (Wang et al. 1996; Abedon et al. 2001), although decoupling host growth rate and density precludes these calculations from predicting the dynamics of the system. Dynamic models have attempted to include plasticity using density-dependent lysis rates instead of latent periods (Weitz and Dushoff 2008), different fixed trait values for different fixed host growth rates (Middelboe 2000), phenomenological expressions (Edwards and Steward 2018), or case-specific effective expressions (Rabinovitch et al. 2002) aimed at improving the design of one-step growth experiments (Aviram et al. 2015).

In spite of the experimental and theoretical work above, how the host growth rate affects the ecological and evolutionary dynamics of the phage-host system remains largely unknown. Here we aim to fill this knowledge gap by addressing the following questions: How does viral plasticity affect the eclipse period, maturation rate, latent period, and burst size in the short and the long terms? How do these trait changes affect the ecological interaction between host and phage in static and dynamic environments? To answer these questions, we focus here on T-phage infecting Escherichia coli, one of the most common host-phage model systems. We first compiled the available data on how T-phage traits change with host performance, to deduce and assess the generality of our own functional forms linking host growth rate with eclipse period and maturation rate. Because the factors that trigger lysis are unknown, we assumed that latent period and associated burst size are evolutionary outcomes. We studied such emerging evolutionary strategies by embedding the datadeduced functions in a standard host-phage mathematical model, which allowed us to study the short- and long-term ecological and evolutionary behaviors of the system. Our results shed light on the mechanisms underlying the timing of lysis under a diversity of environmental conditions, including steady states and dynamic nutrient changes, and how plasticity affects such timing and burst size. Our expressions can help improve the predictability of host-phage models, from smallscale to earth-system models. Moreover, our findings have the potential to be generalized to any lytic phage, including viruses that affect biofilms or are used for phage therapy or industrial setups, and to motivate novel experiments aimed at characterizing viral plasticity across systems.

\section{Methods \\ Data}

We compiled data from one-step growth experiments (Ellis and Delbrück 1939) that measured a comprehensive 
set of viral trait values for various host growth rates (Hadas et al. 1997; You et al. 2002; Birch et al. 2012; Golec et al. 2014; see table 1). Specifically, these experiments followed one infection cycle of Escherichia coli by either the T7 phage (You et al. 2002; Birch et al. 2012) or the T4 phage (Hadas et al. 1997; Golec et al. 2014), both obligately lytic. All the experiments used chemostats in which host growth rates were regulated by using either different sources of carbon (Hadas et al. 1997; Birch et al. 2012) or different dilution rates (You et al. 2002; Golec et al. 2014); culture and infection temperatures were kept to $30^{\circ} \mathrm{C}$ in You et al. (2002), whereas the rest used $37^{\circ} \mathrm{C}$ (see detailed description in app. A; apps. A-J are available online). In all cases, the eclipse period, $E$, and maturation rate, $M$, were reported or the original experimental data were provided. The latter allowed us to estimate $E$ and $M$ consistently across examples by using standard definitions (time after infection at which the first virion is assembled and rate of increase of the chloroformgenerated virion data, respectively).

\section{Data Analysis}

We fitted the extracted eclipse period and maturation rate data as a function of the host growth rate, $\mu$, to obtain functions that include plasticity, $E(\mu)$ and $M(\mu)$. To facilitate comparison across experiments, we normalized $\mu$ using the maximum growth rate $\left(\mu_{\max }\right)$ reported in each experiment for host optimal conditions (compatible with tabulated values for $E$. coli maximum growth rates at the temperature used in each case; Herendeen et al. 1979). For the fits, we focused on simple functional forms that can replicate the data across data sets and are biologically meaningful. Decreasing exponentials, for example, match qualitatively the reported negative correlation with host growth rate reaching a lower plateau for high $\mu$ and have a reduced number of parameters. As shown in figures 1, A1-A3 (left panels), and table B1 (available online), the data available from all our sources are separately compatible with

$$
E(\mu)=E_{\infty}+E_{0} e^{-\alpha_{E} \mu / \mu_{\max }} .
$$

This function captures the high $E$ values observed for very low growth rates, given here by $E_{\infty}$ and $E_{0}$, with $\alpha_{E}$ determining how steeply the function approaches the nonzero minimumvalue plateau. Table B1 shows the parameter values across examples, as well as indicators of the closeness of the function to the data (adjusted $R^{2}$ and root-mean-squared deviation). The maturation rate data show an early slow growth that accelerates for intermediate growth rates and, ultimately, reaches a plateau (figs. 1, A1-A3, right panels), thus resembling a sigmoid function, such as

$$
M(\mu)=\frac{M_{\infty}}{1+e^{-\alpha_{M}\left(\mu / \mu_{\max }-M_{0}\right)}},
$$

where $M_{\infty}$ represents the upper plateau, $\alpha_{M}$ how steeply $M$ reaches it, and $M_{0}$ the midpoint of the function. Although other qualitatively similar functions may also fit the data (see app. B), our results are robust against the specific choice for $E(\mu)$ and $M(\mu)$. The sources of variation across experiments, that is, different T-phages and temperatures, are encoded in equations (1) and (2) via the various coefficients (tables 1, B1). When parametrizing our model, we focused on the You et al. (2002) database (i.e., T7 phage infecting E. coli at $30^{\circ} \mathrm{C}$ ), the most complete data set.

\section{Phage Trait Set}

We use a trait-based approach for the phage in which each viral phenotype is represented by the set of traits defined by the lytic cycle. The adsorption rate, $k$, can be affected by multiple factors not considered here (e.g., host plasticity and evolution), and therefore, we assumed a constant $k$ for all phage phenotypes in our single-host system. Here, $E$ and $M$ were univocally determined by the host growth rate (eqq. [1], [2]). The burst size, $B$, is set by the maturation rate and the time between the end of the eclipse period and lysis, a relationship typically represented with the linear function $B=$ $M[L-E]$ (Wang 2006), where $L$ is the latent period. This expression assumes that only lysis - and not intracellular resources - limits the offspring number. Considering viral plasticity, the burst size can be rewritten as

$$
B(\mu)=M(\mu)[L-E(\mu)] .
$$

The latent period, the only remaining trait, therefore characterizes the viral phenotype (equivalently, the holin gene, re-

Table 1: Compilation of data from the literature

\begin{tabular}{llccl}
\hline Source & \multicolumn{1}{c}{ Host } & Phage & Temperature $\left({ }^{\circ} \mathrm{C}\right)$ & \multicolumn{1}{c}{ Data for fits } \\
\hline You et al. 2002 & Escherichia coli & T7 & 30 & Extracted from original data \\
Birch et al. 2012 & E. coli & T7 & 37 & Extracted from original data \\
Hadas et al. 1997 & E. coli & T4 & 37 & From reported $E$ and $M$ data \\
Golec et al. 2014 & E. coli & T4 & 37 & Extracted from original data \\
\hline
\end{tabular}

Note: The first and last databases used different dilution rates to vary the host growth rate, whereas the central two databases used different sources of carbon. 

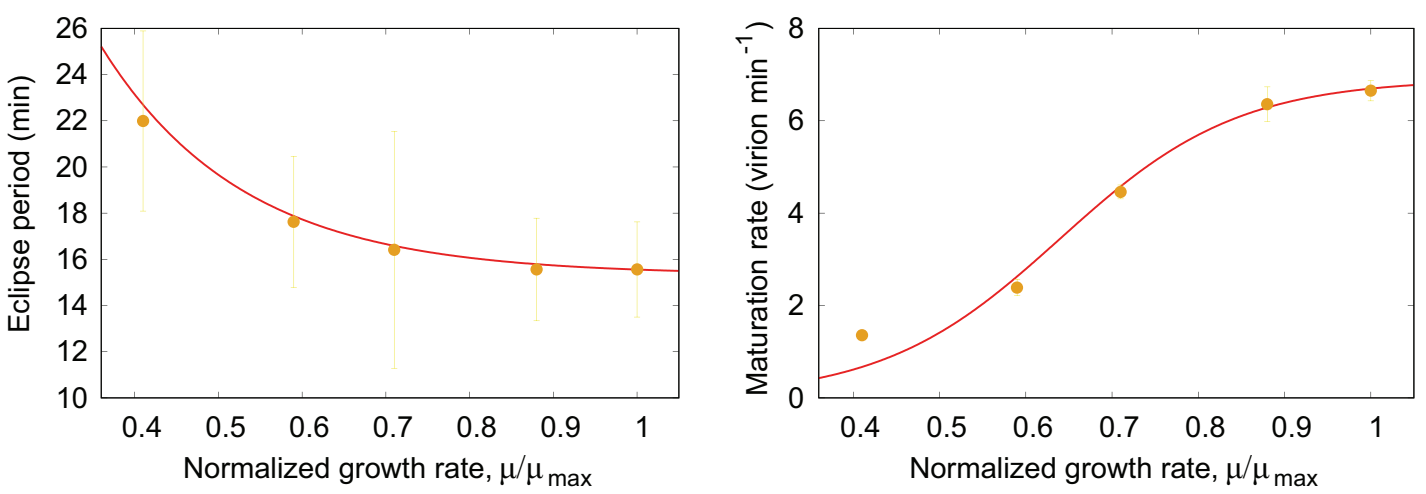

Figure 1: Data for the eclipse period $(E, l e f t)$ and maturation rate $(M$, right $)$ extracted from the one-step growth experiment data in You et al. (2002) as a function of the host growth rate, $\mu$. The error bars represent accumulated error for the extraction method. We have normalized the host growth rate using the maximum growth rate observed in the experiment, $\mu_{\max }=1.7 \mathrm{~h}^{-1}$. The curves represent our proposed functional forms for this case, which fit the experimental data (see table B1).

sponsible for initiating lysis, characterizes genotypes). The exact mechanisms that determine the timing of lysis are unknown, although they correlate with aspects from both phage and host (app. C). Here we assumed that the latent period is shaped by evolution. In the first part of our study (evolutionary analysis; see below), we assumed that $L$ is the only adaptive trait for the phage, influenced by how host quality affects the rest of the traits above; the latent period remains fixed for each genotype, and selection pressures are created by competition and the plasticity in $E$ and $M$. Considering the optimal latent period that emerges from the evolutionary dynamics for different growth rates provides an emergent functional form, $L(\mu)$, that can shed light on how plasticity influences such a trait. We used this result in the second part of our study (dynamic response to perturbations; see below), which considers phenotypes in which $L=L(\mu)$ (together with $E(\mu), M(\mu)$, and $B(\mu)$ ), thus allowing us to explore how a fully plastic virus influences the dynamics of the system.

\section{Theoretical Model}

To represent the ecological dynamics of the host-phage system, we used a classic model that includes explicitly the delay between infection and lysis (Levin et al. 1977). This model has proven to be realistic from both the ecological and evolutionary points of view (Bonachela and Levin 2014) and keeps track of the dynamics of free host cells, $[C]$, infected hosts, $[I]$, free viruses, $[V]$, and nutrient concentration, $[N]$, using the following delayed differential equations:

$$
\begin{aligned}
& \frac{d[C](t)}{d t}=w_{\text {in }}\left[C_{0}\right]+\mu([N])[C]-k[C][V]-w_{\text {out }}[C], \\
& \frac{d[I](t)}{d t}=k[C][V]-\left(k[C]_{t-L}[V]_{t-L}\right) e^{-w_{\text {out }} L}-w_{\text {out }}[I],
\end{aligned}
$$

$$
\begin{aligned}
\frac{d[V](t)}{d t}= & \left(B k[C]_{t-L}[V]_{t-L}\right) e^{-w_{\text {out } L}}-k[C][V]-m[V] \\
& -w_{\text {out }}[V] \\
\frac{d[N](t)}{d t}= & w_{\text {in }}\left[N_{0}\right]-w_{\text {out }}[N]-\frac{\mu([N])}{Y}[C]
\end{aligned}
$$

(see symbols and units in table 2). We emulate here a twostage chemostat, a setup in which a first chemostat allows for a virus-free culture of the host to reach a controlled stationary density, $\left[C_{0}\right]$, which feeds the second chemostat, where the interaction with the phage, equations (4)-(6), happens (Husimi et al. 1982). Such a setup reduces significantly the region of the parameter space for which the typical (predatory-prey-like) oscillations are expected and reduces evolutionary pressures on the host, thus allowing us to focus on the evolution of the phage only. The inflow of fresh hosts at a rate $w_{\text {in }}$ is represented in the first term of equation (4); the second term represents host reproduction, whereas the third term represents new viral attachments and infections. The last term represents the host removal at a rate $w_{\text {out }}$ during dilution events in the chemostat. New infections increase $[I]$ (first term in eq. [5]), which can disappear during dilution (last term) or due to the lysis of cells that became infected exactly one latent period in the past (second term, where $e^{-w_{\text {out } L}}$ is the survival probability for infected cells during the extent of the latent period). The virions released by such cells at lysis contribute to the pool of free phages (first term in eq. [6]), which, in turn, can disappear after infecting free hosts (second term) or due to natural mortality or dilution (last two terms). Finally, the dynamics of the nutrient concentration depend on the nutrient introduced in the second chemostat or removed during dilution (first and second terms) and the assimilation of nutrient by the host (third 
Table 2: Symbols for variables used in the model and parameter values

\begin{tabular}{|c|c|c|c|}
\hline Symbol & Description & Units & Value \\
\hline \multicolumn{4}{|c|}{$\begin{array}{l}\text { Ecological/evolutionary } \\
\text { variables: }\end{array}$} \\
\hline$[N](t)$ & Dissolved inorganic nitrogen concentration & $\mathrm{mol} \mathrm{L}-1$ & Ecological variable \\
\hline$[C](t)$ & Noninfected host concentration & cell $\mathrm{L}^{-1}$ & Ecological variable \\
\hline$[I](t)$ & Infected host concentration & cell $\mathrm{L}^{-1}$ & Ecological variable \\
\hline$[V](t)$ & Free virus concentration & cell $\mathrm{L}^{-1}$ & Ecological variable \\
\hline$M(\mu)$ & Maturation rate & virions $\mathrm{d}^{-1}$ & Eq. (2); table B1 \\
\hline$E(\mu)$ & Eclipse period & d & Eq. (1); table B1 \\
\hline$L$ & Latent period & $\mathrm{d}$ & Evolutionary variable \\
\hline$B$ & Burst size & virions & Evolutionary variable \\
\hline$\mu_{v}(t)$ & Virus population growth rate & $\mathrm{d}^{-1}$ & Ecological variable \\
\hline$\mu(t)$ & Host population growth rate & $d^{-1}$ & Ecological variable \\
\hline \multicolumn{4}{|c|}{ Fixed parameters: } \\
\hline$\mu_{\max }$ & Maximum host population growth rate & $\mathrm{d}^{-1}$ & 40.8 \\
\hline$Y$ & Yield parameter & cell mol $\mathrm{m}^{-1}$ & $9 \times 10^{13}$ \\
\hline$K_{N}$ & Half-saturation constant for growth & mol L ${ }^{-1}$ & $10^{-6}$ to $2 \times 10^{-4}$ \\
\hline$k$ & Adsorption rate & 1 cell $^{-1} \mathrm{~d}^{-1}$ & $9 \times 10^{-10}$ \\
\hline$m$ & Virus mortality rate & $\mathrm{d}^{-1}$ & .09 \\
\hline$p_{m}$ & Virus mutation probability & $\ldots$ & $10^{-8}$ to $10^{-6}$ \\
\hline$r_{i}$ & Virus invasion rate & $\mathrm{d}^{-1}$ & $10^{-2}$ to 1 \\
\hline$\left[C_{0}\right]$ & Noninfected host supply concentration & cell $\mathrm{L}^{-1}$ & $0 ; 10^{8}$ \\
\hline$\left[N_{0}\right]$ & Dissolved inorganic nutrient supply concentration & $\mathrm{mol} \mathrm{L} \mathrm{L}^{-1}$ & 45 to $2,000 \times 10^{-6}$ \\
\hline$w_{\text {out }}$ & Chemostat dilution rate & $\mathrm{d}^{-1}$ & .24 to 21.84 \\
\hline$\left[N_{\text {ext }}\right]$ & External source for dissolved inorganic nutrient & $\mathrm{mol} \mathrm{L}-1$ & Fixed with $\left[N_{0}\right]=\left[N_{1 s t}\right]+\left[N_{\text {ext }}\right]$ \\
\hline$\left[N_{1 \mathrm{st}}\right]$ & Dissolved inorganic nutrient in first chemostat & $\mathrm{mol} \mathrm{L}^{-1}$ & Fixed with eq. (E1) \\
\hline$\left[N_{0_{1 \mathrm{st}}}\right]$ & $\begin{array}{l}\text { Dissolved inorganic nutrient supply in first } \\
\text { chemostat }\end{array}$ & $\mathrm{mol} \mathrm{L}^{-1}$ & Fixed with eq. (E2) \\
\hline$w_{\text {in }}$ & Chemostat inflow rate & $d^{-1}$ & $w_{\text {out }}$ \\
\hline
\end{tabular}

Note: Data for the host obtained from Fagerbakke et al. (1996), Herendeen et al. (1979), and Schulze and Lipe (1964); data for the virus into the ranges shown/used in Bull et al. (2006), De Paepe and Taddei (2006), Shao and Wang (2008), and Weitz et al. (2005). For the calculation of the yield factor from the references, we assumed a fixed carbon content per host cell of $10^{-12} \mathrm{~g}$.

term). We assumed that the growth rate for free hosts can be described by the simple Monod model (Monod 1950), $\mu=$ $\mu_{\max }[N] /\left([N]+K_{N}\right)$, where $\mu_{\max }$ is the maximum growth rate and $K_{N}$ is the half-saturation constant for growth (inversely correlated with growth affinity). See figure 2 and appendixes $\mathrm{C}-\mathrm{E}$ for further details and assumptions.

\section{Analytical Approach}

To obtain an initial understanding about the effect of viral plasticity on the long-term behavior of the system above, we analyzed its ecological and evolutionary stationary states. For the ecological stationary states, we calculated the solutions to the equations $d[C] / d t=d[I] / d t=d[V] / d t=$ $d[N] / d t=0$ and studied the associated stability (see apps. C-E). For the evolutionary stationary state, we conducted invasion analyses by studying the stability of a phage-host system that, after reaching equilibrium, is inoculated with an invading viral phenotype (i.e., viral strain with a different $L$ value) that challenges the resident phage. Such invaders can also represent new genotypes that result from mutations in the holin gene and therefore show different latent periods. The invasion analysis determines the phenotype (i.e., $L$ ) that maximizes fitness, the evolutionarily stable strategy (ESS; Geritz et al. 1998; Dercole and Rinaldi 2008). The ESS can be identified as the phenotype that will dominate the system in the long term even in the presence of all possible variability. From the $L_{\mathrm{ESS}}$ resulting for different host growth rates, we composed a first approximation to the plasticity curve for the latent period, $L(\mu)$. See appendix F for further details.

\section{Numerical Approach}

The analytical approach above requires important assumptions such as the quick relaxation to the ecological equilibrium before mutants enter the system, which risks overlooking the dynamic influence of plasticity. Under realistic 


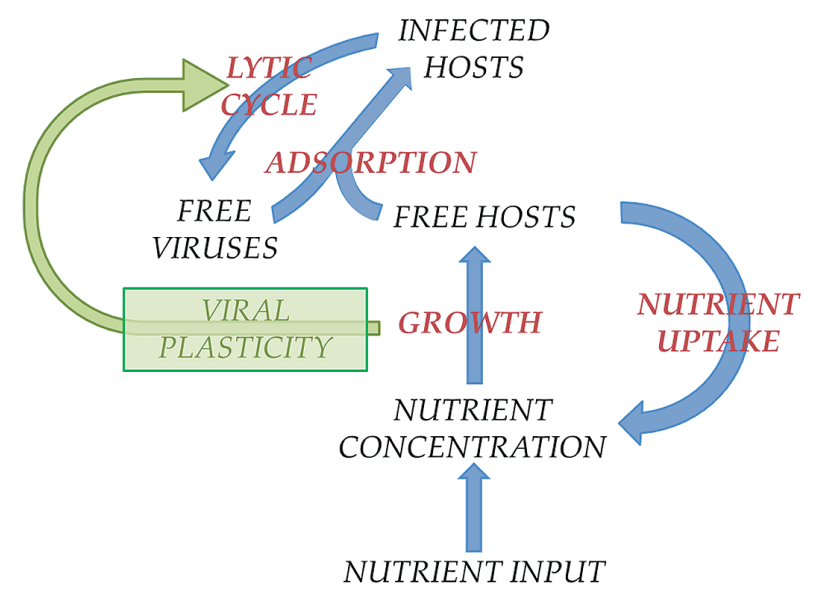

Figure 2: Flow diagram showing the interactions for host, phage, and environment as described by equations (4)-(6) (blue arrows) and the role of viral plasticity (green arrows). Black type is used for the interacting agents in the system (i.e., dynamic variables of the model), whereas red type represents processes.

conditions, plasticity and evolution can interact nontrivially, which may in turn feed back to the ecological dynamics of host and phage (Lennon and Martiny 2008). Such dynamic interaction, however, precludes the analytical calculation of a closed expression for the ESS. To go beyond such constraints, we used a numerical eco-evolutionary simulation framework in which both ecology and evolution occur at the same timescale (for details, see Menge and Weitz 2009; Bonachela and Levin 2014; Lomas et al. 2014; app. H). Starting from a single viral phenotype and host populations, equations (1)-(7) are integrated, thus providing the ecological dynamics of the system. Mutants originate from parental phenotypes, at random times, by means of a genetic algorithm in which the phenotype with the highest probability to be selected for mutation is the one with the highest relative abundance in the system (Menge and Weitz 2009). Thus, multiple resident and mutant phenotype populations compete for the single host at any given time. Mutation and natural selection facilitate an alternation of dominance until, eventually, a phenotype rises that cannot be challenged by any other mutant. Such a strategy (the ESS) can be identified as an evolutionary stationary state in the representation of the dominant $L$ against time. Similarly to the analytical work above, although the simulations assume a fixed $L$ for each phenotype, compiling the latent periods that dominate for each different host growth rate may reveal how selection has cast the dependencies of a plastic lysis timing, $L(\mu)$. Motivated by the viral plasticity experiments collated here, we varied the host growth rate using different (i) input concentrations, $\left[N_{0}\right]$; (ii) sources of sugar, which we emulated by using a variety of growth affinities (i.e., $K_{N}$ ); or (iii) dilution rates, $w_{\text {out }}$. We also explored the evolutionary outcome of scenarios away from stationarity. To this end, we repeated the experiments above implementing rapid environmental variation through an input concentration that varied on a daily basis by a random factor up to $\sigma$, with $\sigma=0.3$ and $\sigma=0.5$.

In all cases, to quantify the trait ranges enabled by plasticity, we normalized the resulting $L(\mu)$ and $B(\mu)$ curves by their minimum value. Such normalization also facilitates comparison across the methods described above to control $\mu$, with the ESS predicted by a nonplastic version of the model $\left(L_{\text {non }}\right.$ and $\left.B_{\text {non }}\right)$. Because nonplastic models use fixed trait values typically obtained from available experiments, which standardly set optimal growth conditions for the host, we assumed for this comparison that $L_{\text {non }}$ and $B_{\text {non }}$ correspond to the $L$ and $B$ values obtained for the maximum $\mu$ observed in each simulation (see app. F).

A last in silico experiment gauged how phage plasticity alters predictions in diverse dynamic scenarios with sudden changes in nutrient availability. We used the two-stage chemostat represented by equations (1)-(7), with a baseline nutrient input concentration that sets the host growth rate to approximately half of its maximum. We explored three consecutive events: a sustained nutrient pulse that takes the host to its maximum growth rate and another that takes the host to two-thirds of its maximum, followed by a reduction in the input concentration that decreases host growth to one-third of its maximum. We fixed the duration of each event to $20 \mathrm{~d}$, separated by resting periods of $20 \mathrm{~d}$. With this setup, we compared the predictions of three different versions of the model above: (i) one that ignores phages (mimicking most current ecosystem models); (ii) a nonplastic description of the phage (like standard host-phage models, i.e., fixed viral traits obtained by assuming optimal conditions for the host); and (iii) our plastic description. For the latter, we used the expression for the plastic latent period, $L(\mu)$, and burst size, $B(\mu)$, deduced from the eco-evolutionary simulations, which allowed us to include a fully plastic virus (i.e., all four traits respond to changes in the host) under the assumption that it is the most dominant/representative phenotype of the focal region of the ecosystem.

Finally, we tried all the experiments above with one-stage chemostats (i.e., no fresh hosts or nutrient supplied by the first chemostat; app. E) as well to test the role of the marked oscillations typically observed in such cases.

\section{Results}

\section{Analytical Results}

Two-stage chemostats facilitate both ecological and evolutionary stationarity in our system. The former provides a nontrivial ecological stationary state in which both host and viral population coexist, given by equations (D1)-(D3). As 
described in appendix $\mathrm{F}$, the latter leads to an associated evolutionary stationary state (i.e., the ESS) for the phage that changes with the host growth rate:

$$
L(\mu)=\frac{1}{w_{\text {out }}}+E(\mu)
$$

where $w_{\text {out }}$ is contributed by the exponential term in equations (5) and (6) and, therefore, represents more generally the removal rate for intracellular virions (equivalently, infected hosts). Equation (8), together with the trade-off function, equation (3), provides the associated burst size,

$$
B(\mu)=\frac{M(\mu)}{w_{\text {out }}} .
$$

This ESS maximizes the viral fitness and minimizes the amount of hosts (i.e., resources) the phage needs, the expected outcome of classic competition theory (Tilman 1982). Note that the expressions for both the ecological and evolutionary stationary states are valid regardless of the specific details of $E(\mu)$ and $M(\mu)$.

\section{Numerical Results}

The impossibility of finding a closed form for the host growth rate as a function of time and the constraints of the analytical calculations required the use of our ecoevolutionary simulations for the diversity of scenarios below. Still, informing the analytical ESS with the numerical host growth rate provided a reference curve to compare with the numerics. For all our simulations, we parametrized the model using table 2 and the You et al. (2002) column from table B1. Following standard practice for chemostats, we set $w_{\text {in }}=w_{\text {out }}$, although for coherence with the analyt-

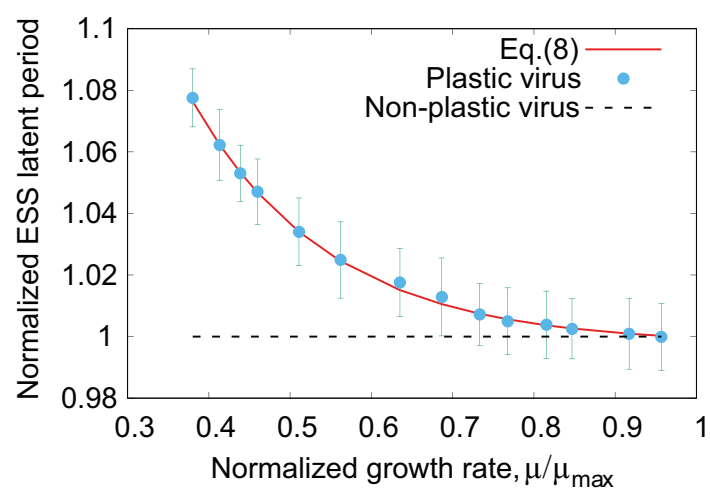

ical calculations, we will keep referring to the dilution rate as $w_{\text {out }}$.

Constructing the $\mathrm{L}(\mu)$ Curve: Long-Term Behavior for Different Host Growth Rates. The ecological interactions including plasticity (eqq. [4]-[7] with eqq. [1]-[3]), together with evolution, give rise to trait dynamics such as those shown in figure F1 (right panel). Although the figure shows only the most dominant phage type, many different phenotypes compete for the same host at any given time, ensuring a wide trait distribution. Mutation and selection thus enable the exploration of the phenotypic space until, eventually, a phenotype emerges to overwhelmingly dominate over any new or existing phenotypes and the system reaches an evolutionary stationary state. Because this exploration is intrinsically stochastic, the evolutionary stationary state values resulting from each replicate are spread around a mean value that we define as $L_{\mathrm{ESS}}$. Here we used 300 replicates for each example.

We first studied the $L_{\mathrm{ESS}}$ emerging from such evolutionary dynamics using $\left[N_{0}\right]$ to control the host growth rate. Using input concentrations that ranged from $\left[N_{0}\right]=7 \times$ $10^{-5} \mathrm{~mol} \mathrm{~L}^{-1}$ to $\left[N_{0}\right]=2 \times 10^{-3} \mathrm{~mol} \mathrm{~L}-1$ and fixing the dilution rate to $w_{\text {out }}=15 \mathrm{~d}^{-1}$, the stationary host growth rate ranged from $\sim 15.5 \mathrm{~d}^{-1}$ to $\sim 39.0 \mathrm{~d}^{-1}$, respectively (in fig. 3, expressed as fractions of $\mu_{\max }$ ). Thus, there is a positive correlation between the host growth rate and nutrient input concentration that cannot be inferred easily from the stationary state equations. For reference, figure 3 shows also the curve obtained using equation (8) (similarly for the burst size using eq. [9]). The maximum range of variation for $L_{\mathrm{ESS}}$ is $\sim 8 \%$, while there is an $\sim 18$-fold range for $B_{\mathrm{ESS}}$. The $L_{\mathrm{ESS}}$ values decrease and the $B_{\mathrm{ESS}}$ values increase with host growth rate, opposite to the usual positive correlation be-

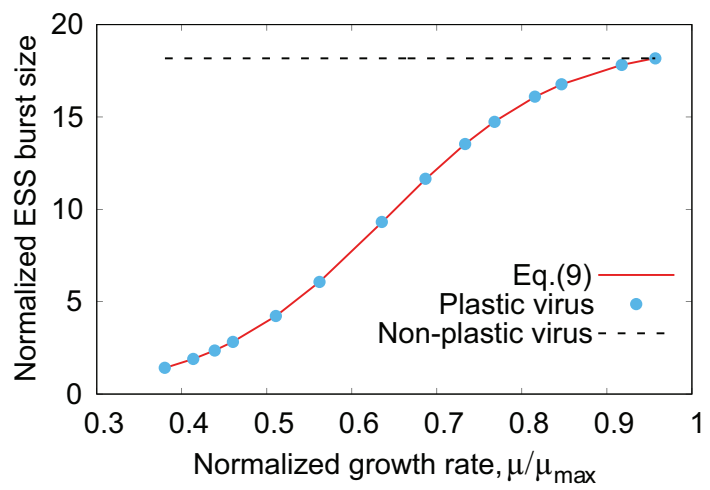

Figure 3: Evolutionary results from theory (solid line) and averaging over simulations (points) obtained when altering the host growth rate using $\left[N_{0}\right]=7 \times 10^{-5} \mathrm{~mol} \mathrm{~L}^{-1}$ to $\left[N_{0}\right]=2 \times 10^{-3} \mathrm{~mol} \mathrm{~L}^{-1}$, with $w_{\text {out }}=15 \mathrm{~d}^{-1}$ and $K_{N}=9 \times 10^{-5} \mathrm{~mol} \mathrm{~L}^{-1}$. The whiskers in all simulation results represent the standard deviation across replicates, and the dashed line represents the value that typical models ignoring plasticity would use. Both plastic curves and data have been normalized using the minimum value obtained in the simulations, while the nonplastic description shows the $\mu_{\max }$ value for the traits. Left, latent period, L. Right, burst size, $B$. ESS $=$ evolutionarily stable strategy. 
tween lysis time and offspring number (fig. J2, left). There is, thus, an improvement in phage performance with host growth rate, as increasing $\left[N_{0}\right]$ (i.e., $\mu$ ) leads to an increased concentration of viral individuals and a consequent decrease in the concentration of hosts (fig. J2, right). In consequence, $L_{\mathrm{ESS}}$ increases with the stationary concentration of free hosts, $[C]_{\text {st }}$ (fig. J3, left), while the number of infected hosts shows a nonmonotonic behavior but low variability (fig. J3, right). The typical nonplastic description for the system shows the minimum possible $L_{\mathrm{ESS}}$ and maximum possible $B_{\mathrm{ESS}}$ within the range of variation of these traits, increasing phage and infected individual densities, but a number of free hosts that does not depend on the host growth rate (dashed lines in figs. 3, J2, J3; app. F).

We observed similar results when varying the host growth rate using different $K_{N}$ values as a proxy for different sources of carbon (fig. J4, left). When using the dilution rate as a way to vary the host growth rate, on the other hand, plasticity played a negligible role. In two-stage chemostats, host growth rate and dilution rate are negatively correlated (illustrated theoretically in Bonachela and Levin 2014); in our case, however, $[N]$ shows an approximately constant value for any $w_{\text {out }}$ (see app. G), which translates into a very limited range of variation for $\mu$. As a result, varying $w_{\text {out }}$ affects mostly the denominator in equations (8) and (9), and $L$ and $B$ show a behavior similar to that of the nonplastic description, with the classic positive correlation between both traits that sets an evolutionary trade-off between generation time and offspring (fig. 4; fig. J4, right). The freehost availability decreases with the (subtle) variation in $\mu$.

The Role of Fluctuations in the ESS. We repeated the three methods above with classic one-stage chemostats (i.e., assuming no inflow from the first chemostat; see app. E). We observed qualitatively similar trends as above: a $L_{\mathrm{ESS}}$ that decreases and a $B_{\mathrm{ESS}}$ that increases with host growth rate (fig. 5),

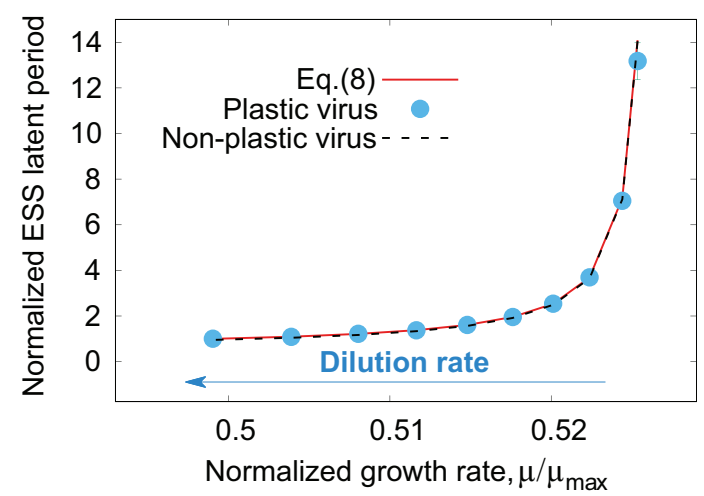

with the exception of the positive correlation between burst size and latent period obtained when using $w_{\text {out }}$ to control $\mu$. When an equilibrium was reached (in fig. 5, range $\left.\mu / \mu_{\max } \sim 0.25-0.35\right)$, the stationary state was accurately described by equations (D1)-(D3), and in consequence, the ESS was well described by our analytical expressions (solid lines, resulting from eqq. [8], [9] informed by the numerical growth rate). These expressions, however, failed to describe the wide oscillations found for the rest of the growth rates $\left(\mu / \mu_{\max }>0.35\right)$, for which a steeper decrease for $L(\mu)$ and a milder increase for $B(\mu)$ were observed. The range of the emergent $L_{\mathrm{ESS}}$ increased with respect to the theoretical prediction and that of $B_{\mathrm{ESS}}$ decreased. On the other hand, fluctuations introduced in two-stage chemostats by means of a random (daily) forcing of the input concentration did not lead to significant departures from the analytical expression (fig. H1).

Short-Term Behavior under Dynamic Environmental Conditions. Finally, we shifted the focus to short-term population dynamics and compared the predictions of three different versions of the model for various dynamic events. The model without viruses shows a host population that follows the qualitative behavior of the nutrient input (see figs. 6, J5). For the nonplastic description, the phage and infected host populations show the qualitative profile of the nutrient input as a response to the attempts of the (free) host population to cope with nutrient changes. However, the phage ecological pressure maintains the average host population around approximately the baseline level throughout the whole experiment. Different (fixed) phage parameterizations only altered the result quantitatively and, in some cases, introduced an oscillatory behavior for the different nutrient regimes. With our plastic description for all four viral traits, counterintuitively, events that increase the host growth rate lead to a decrease in the free host population with respect to

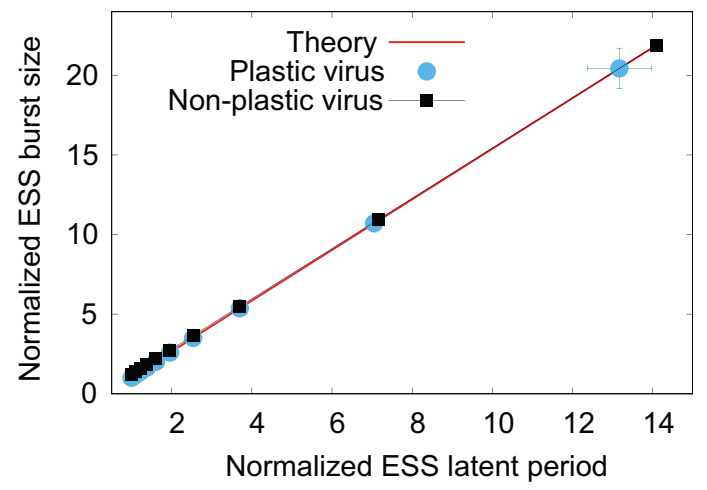

Figure 4: Evolutionarily stable strategy (ESS) obtained modifying the growth rate through $w_{\text {out }}=0.1 \mathrm{~d}^{-1}$ to $w_{\text {out }}=0.91 \mathrm{~d}^{-1}$, with $\left[N_{0}\right]=1 \times 10^{-4} \mathrm{~mol} \mathrm{~L}^{-1}$ and $K_{N}=9 \times 10^{-5} \mathrm{~mol} \mathrm{~L}^{-1}$. Left, emerging latent period as a function of host growth rate. Right, burst size as a function of latent period, resembling the classic trade-off between the two traits. 

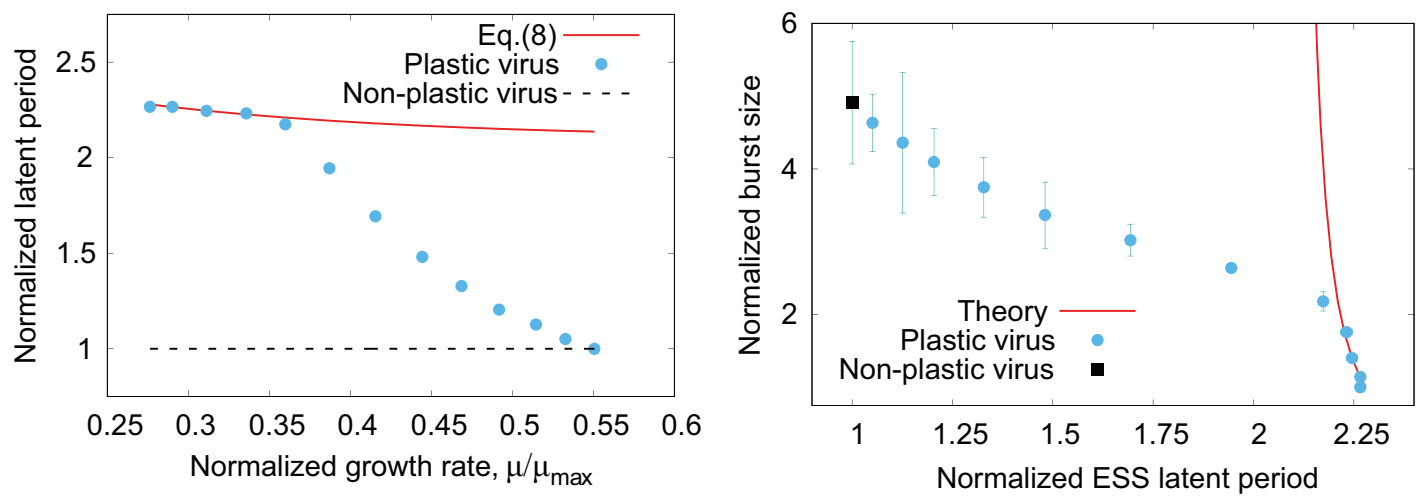

Figure 5: Curves obtained when host growth rate is varied by changing $\left[N_{0}\right]$ in one single chemostat for the interaction phage-host (i.e., limit $\left.\left[C_{0}\right]=\left[N_{1 s t}\right]=0\right)$ with $w_{\text {out }}=0.2 \mu_{\max }=8.16 \mathrm{~d}^{-1}$. Left, emerging latent period as a function of host growth rate. Right, emerging burst size as a function of the latent period. Note that the points represent averages over the final hundreds of days of the dynamics to account for possible oscillations. The points that depart from predictions correspond to the cases for which oscillations were observed. ESS $=$ evolutionarily stable strategy.

the baseline, linked to an increase in phage population (figs. 6, $\mathrm{J} 5$, right). Also against intuition, nutrient scarcity leads to an increase in the host population and a decrease of the phage population. The qualitative behavior of the host population thus radically differs across descriptions. Plastic and nonplastic phage behaviors agree qualitatively and quantitatively only when $\mu=\mu_{\max }$ in the case shown in figure J5. We repeated the same experiment considering remineralization, which did not change qualitatively the described behavior (app. I). On the other hand, it is possible to find population oscillations also with the plastic description.

Oscillations in Short-Term Behavior. The three-regime in silico experiment above typically produced a succession of stationary states in two-stage chemostats (fig. 6), with

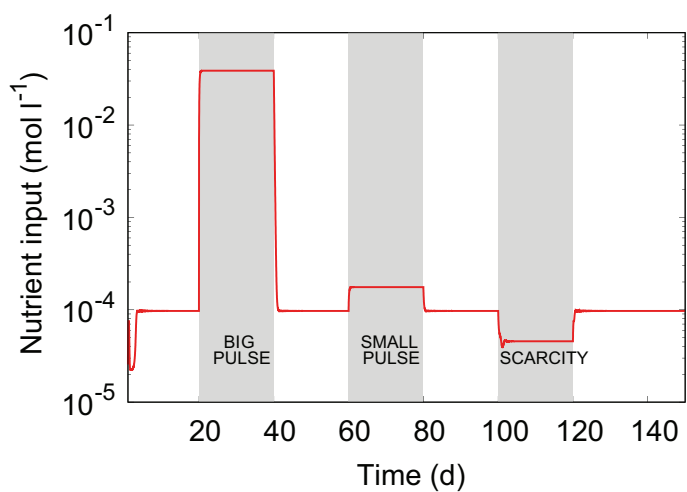

changes in the nutrient input marking the transition between those states. Phage acclimation (via plasticity in $E$, $M, B$, and $L$ ) translated generically into damped oscillations when transitioning between regimes. Similarly to the evolutionary case, however, classic chemostats almost generically led to sustained oscillations. In such cases, both nonplastic and plastic descriptions show a qualitatively similar behavior when looking at averages within each of the distinct regimes. However, the amplitude and period of the oscillations are greatly increased in the plastic case for the pulse regimes and decreased for the starvation regime (fig. J6). Moreover, the changes in amplitude in the plastic case are mostly localized, stretching downward the valleys of the host and phage oscillations, but upward the peaks for $[V]$ only. The amplitude and oscillations in $[N]$ and $\mu$ remain similar for both descriptions.

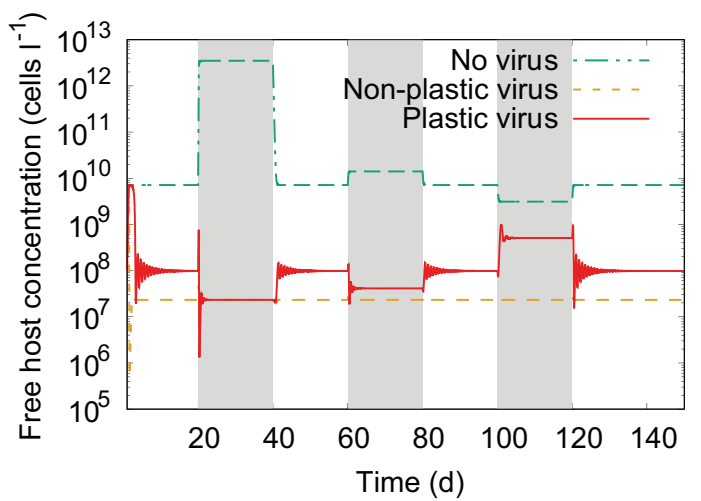

Figure 6: Dynamics of one replicate of the two-chemostat setup for three different events (shaded areas) in which the nutrient inflow brings the host growth rate, respectively, to $\mu=\mu_{\max }, \mu=2 \mu_{\max } / 3$, and $\mu=\mu_{\max } / 3$, comparing a version of the model with no viruses to another one with nonplastic viruses and our plastic description. Left, nutrient profile. Right, available hosts. 


\section{Discussion}

The lytic reproductive cycle and associated viral traits depend to a great extent on the host. The experimental information available does not provide any indication as to what extent the reported changes in $E, M, B$, and $L$ are an active response of the virus or a passive consequence of the physiological changes in the host. In this regard, using the term "viral plasticity" can seem confusing. However, the term summarizes the fact that such traits change with environmental conditions (the host) without any underlying genetic change in the virus. For this reason, it has been used in the past in the same context (Abedon et al. 2001), and we use it here, regardless of whether the plastic response is driven by the host or virus (or both).

We have compiled available data on how T-phage eclipse period and maturation rate change with host growth rate to study analytically and computationally the effects that such viral phenotypic plasticity has on phage traits and phage and host populations. To this end, we have obtained the $L(\mu)$ curve from the optimal lysis timing emerging under various growth conditions that, together with the eclipse period and maturation rate, determines $B(\mu)$. Thus, our results show that plasticity affects all four traits.

\section{Generic Mechanisms and Functional Forms for Phage Plasticity}

The functional forms for $E(\mu)$ and $M(\mu)$ we introduced here (decreasing exponential function with a nonnegligible minimum plateau and standard logistic function, respectively) are consistently compatible with the data obtained for different T-phages under different experimental conditions. Both qualitative forms are biologically meaningful. An increase in host growth rate correlates with an increase in the transcription and translation machinery and rates (as would happen in a healthy host), thus leading to the initial decline in the time needed to synthesize viral components shown by $E(\mu)$. The lower plateau may relate to physiological limits to such synthesis, for example, limits to host metabolic rates (Birch et al. 2012), limits to viral DNA injection time, or translation limitation due to emerging bottlenecks (You et al. 2002). For similar reasons, increasing host growth rates materialize in a quick improvement for $M(\mu)$, slowing down and eventually being limited at high growth rates by ribosome efficiency, polyribosome elongation rate, and late protein expression (You et al. 2002; Calendar and Abedon 2006). This biological interpretation of the proposed functions is supported by how the curves change for different phage strains and temperatures across databases (app. B).

Note that the mechanisms mentioned above are a fundamental part of the replication of most lytic phages, which suggests that these or qualitatively similar curves may de- scribe, generically, phage plasticity. Phage-specific details and environmental factors such as temperature are encoded in the parameters of these functions (table B1). On the other hand, because all the compiled data share the same host species, we cannot discern whether the host influences any parameter other than the normalization factor $\mu_{\max }$, which we used naively to deal with temperature-related and host-strain-specific differences. Nonetheless, our $L(\mu)$ and $B(\mu)$ curves do not depend on the shape of the $E(\mu)$ and $M(\mu)$ functions. Exhaustive experiments aiming at gathering information on different phages and hosts are needed in order to assess the generality of the functional forms and predictions presented here.

In support of this qualitative generality is the fact that the vast majority of the experimental work mentioned above reports decreasing latent periods as growth rate increases, resembling mildly declining exponentials with a nonzero lower plateau, whereas burst sizes increase rapidly. The degree of plasticity for $B$ is significantly more marked than that for $L$ (e.g., Webb et al. [1982], who report a 18-fold variation in $B$ across different viral strains as $\mu$ increases). In our model, we have observed such behavior under stationarity, with and without associated stochasticity and oscillations, which shows the robustness of these results across environmental conditions.

\section{The Mechanisms Underlying the Observed Latent Periods, Burst Sizes, and Trade-Offs}

The plasticity expressions deduced here shed some light on the mechanisms underlying the plastic behavior of these traits. In our system, the emerging latent period results from the interaction between extracellular factors and the use of the host's (intracellular) resources, specifically between the timing for infected-cell removal (the $w_{\text {out }}^{-1}$ term) and plasticity in synthesis time (encoded in $E(\mu)$ ). The former shapes $L$ across generations and has the potential to change the range of variation of $L(\mu)$ with respect to that of $E(\mu)$. When the dilution rate, $w_{\text {out }}$, is fixed to sufficiently small values, the constant term dominates over that of $E(\mu)$, which would lead to the mild effect of plasticity on $L$ reported here across examples and in experiments. Higher $w_{\text {out }}$, however, results in a more noticeable plasticity for $L$, especially in the low $\mu$ limit. Low growth rates, in this case, match high $[C]_{\mathrm{st}}$, thus resonating with past theories about the increasing role of plasticity in $L$ as host density increases (Wang et al. 1996). For the oscillatory cases, the range of variation for $L$ is much amplified, but unfortunately, our expressions fail to provide an analytical understanding of the underlying mechanisms.

Due to the conspicuous degree of plasticity for the maturation rate, an increase in host performance allows the phage to produce more virions with a shorter lytic cycle, 
unsurprisingly breaking the classic trade-off. Therefore, we hypothesize that the importance of plasticity does not reside in the modification of the generation time but rather in the pronounced modification of the offspring number. On the other hand, when controlling the host growth rate by tuning $w_{\text {out }}$, decreasing the dilution rate barely affects $\mu$, thus rendering plasticity negligible. With negligible plasticity, higher burst sizes can only be achieved with longer latent periods, leading to the classic positive correlation between $L_{\mathrm{ESS}}$ and $B_{\mathrm{ESS}}$ and negative correlation between $L_{\mathrm{ESS}}$ and $[C]_{\text {st }}$. As the survival probability of the latent offspring increases and the abundance of available hosts decreases, it becomes advantageous to lyse hosts at later times, and synthesis time (i.e., E) becomes mostly irrelevant.

\section{Plasticity Inverts Expectations for Short-Term Population Dynamics}

The wide range of possible burst sizes enabled by plasticity affect the ecological dynamics for both host and phage populations, altering dramatically the expectations built with standard models that neglect plasticity. Plasticity affects the strength and timing of the interaction pathways between host, virus, and environment (figs. 2, J1). Infected hosts play a pivotal role in such feedbacks as they result from, and mediate, the interaction between free hosts and phages.

Nutrient pulses like the ones described in the short-term experiments increase $\left[N_{0}\right]$ and, in consequence, host growth rate. In the nonplastic description, the result is an increase in infections that, after a certain period of time $L$, lead to an increase in phage number effectively keeping the density of available hosts unaltered (e.g., baseline $[C]$ does not change following nutrient pulses; see eq. [D1]). The expected host population growth is absorbed into an increased number of infections. Plasticity in $E, M, B$, and $L$, however, enables a higher phage production in less time, leading to an increased phage-induced mortality rate that reduces the number of available hosts and infections needed to sustain a similar viral growth (eqq. [D1]-[D3], applicable within each regime), which exacerbates the "kill the winner" response widely observed in the field and in models (Winter et al. 2010). This boost in phage performance translates into dynamic competition abilities for the phage, thus showcasing plasticity as a facilitator of interactions between phage ecological and evolutionary dynamics. Such modulation is constrained, as the increase in competition abilities resulting from an increasing $\mu$ is limited by the existence of a saturation regime for $M$ and a nonzero lower plateau for $E$. Such physiological limits have been overlooked in the few existing attempts to include plasticity in dynamic models. Nonplastic descriptions are also disadvantaged to describe such dynamic conditions because they use constant values to represent phage traits such as $B$ that can vary widely with host physiology and thus depart from any fixed parametrization.

\section{Plasticity Reinforces Dynamic Feedbacks between Host, Virus, and Environment}

The changes in the timing and strength of the feedbacks described above can be exemplarily observed in our short-term simulations when sustained oscillations are present. For the plastic description, pulses lead to an improved phage performance and an increase in $[I]$ that feed back to increase $[V]$ much beyond that from the nonplastic case. Such a large phage population draws $[C]$ down to densities much below the expectation from nonplastic models, dragging with it, sequentially, the number of infected hosts and free viruses; the reduced number of hosts, in turn, facilitates the recovery of $[N]$, thus reinitializing the cycle. Due to these much deeper oscillation valleys, however, the recovery time for $[C],[I]$, and $[V]$ is significantly increased in this plastic description, which increases the delay among the three curves and consequently increases the oscillation period.

\section{Applicability and Limitations}

Our expressions preclude the use of different parameterizations to capture different locations or environmental conditions for the same host-phage system. Average or specific parameterizations will not be able to account for dynamic changes in host physiology. Considering viral plasticity, thus, can alter nontrivially the predictions of standard models through the dynamic interaction between phage and host performance. For biogeochemical models, such interaction determines key aspects such as primary production. Our plastic expressions for the most dominant phenotype can help improve the predictability of such models, as the two-stage chemostat and inflow and outflow of hosts and nutrients roughly represent a volume of oceanic water. Such flows can also represent those in the human gut, and therefore our expressions can help the study of phages infecting the gastrointestinal tract. As it does for biofilm phages, space will play in these cases a key role, imposing marked resource gradients that emphasize the need to consider plasticity over static parameterizations. The relevance of space, nonetheless, also increases the relevance of $k$, as the time and distance between adsorption events influences the phage strategy. On the other hand, representing the astonishing viral diversity observed in nature is one of the main challenges to including viruses in large-scale models. Plasticity helps include a dynamic phenotypic diversity for the phage community without the need to invoke any genetic change. Accounting for other realistic details - such as host predation mortality (which affects cell removal rates), alternation between lytic and lysogenic infection modes, or a more accurate description of the 
host-requires changing the standard model used here and, therefore, may alter our predictions. Future work will include host plasticity and coevolution, in which aspects such as cell size (which affects the adsorption rate) play an important role. From a more general perspective, our work can also advance knowledge on how prey physiology (e.g., nutritional level) can affect the ecological and evolutionary strategies of predators (e.g., prey selection and/or predatory rates).

As a final remark, a note of caution: Although the qualitative agreement between our $L$ and $B$ and experimental observations shows that our theory includes key underlying mechanisms, experiments measuring plasticity in these traits do not reach stationarity, typically lyse cells artificially, and do not necessarily target evolutionary stationary states. Stationarity is not reached in the oceans or other realistic scenarios, cases for which our numerical framework is more suitable. Ultimately, additional experimental information is required in order to improve and generalize our theory across systems, thus contributing to advancing our understanding of host-phage interactions.

\section{Acknowledgments}

We would like to thank C. Caceres, M. Heath, P. Suthers, J. Weitz, J. Yin, and A. Zaritsky for helpful discussions; E. W. Birch and P. Golec for providing published data for calculations; E. Akçay and two anonymous reviewers for their suggestions, which have greatly improved the manuscript; and Institute Carlos I for Theoretical and Computational Physics at the University of Granada for the use of its computational infrastructure. M.C. and J.A.B. were supported by the Marine Alliance for Science and Technology for Scotland pooling initiative, funded by the Scottish Funding Council (HR09011) and contributing institutions.

\section{Literature Cited}

Abedon, S. T. 1989. Selection for bacteriophage latent period length by bacterial density: a theoretical examination. Microbial Ecology 18:79-88.

-2011. Bacteriophages and biofilms: ecology, phage therapy, plaques. 1st ed. Nova Science, New York.

Abedon, S. T., S. Duffy, and P. E. Turner. 2009. Bacteriophage ecology. Pages 42-57 in M. Schaecter, ed. Encyclopedia of microbiology. Elsevier, Oxford.

Abedon, S. T., T. Herschler, and D. Stopar. 2001. Bacteriophage latentperiod evolution as a response to resource availability. Applied and Environmental Microbiology 67:4233-4241.

Abedon, S. T., P. Hyman, and C. Thomas. 2003. Experimental examination of bacteriophage latent-period evolution as a response to bacterial availability. Applied and Environmental Microbiology 69:7499-7506.

Aviram, I., A. Rabinovitch, and A. Zaritsky. 2015. Maximizing yields of virulent phage: the T4/Escherichia coli system as a test case. Journal of Theoretical Biology 364:428-432.
Beretta, E., and Y. Kuang. 2001. Modeling and analysis of a marine bacteriophage infection with latency period. Nonlinear Analysis: Real World Applications 2:35-74.

Berg, H. C., and E. M. Purcell. 1977. Physics of chemoreception. Biophysical Journal 20:193-219.

Birch, E. W., N. A. Ruggero, and M. W. Covert. 2012. Determining host metabolic limitations on viral replication via integrated modeling and experimental perturbation. PLoS Computational Biology 8: e1002746.

Bonachela, J. A., and S. A. Levin. 2014. Evolutionary comparison between viral lysis rate and latent period. Journal of Theoretical Biology 345:32-42.

Breitbart, M. 2012. Marine viruses: truth or dare. Annual Review of Marine Science 4:425-448.

Brown, C., J. Lawrence, and D. A. Campbell. 2006. Are phytoplankton population density maxima predictable through analysis of host and viral genomic DNA content? Journal of the Marine Biological Association of the United Kingdom 86:491-498.

Bull, J., J. Millstein, J. Orcutt, and H. A. Wichman. 2006. Evolutionary feedback mediated through population density, illustrated with viruses in chemostats. American Naturalist 167:E39-E51.

Calendar, R., and S. T. Abedon, eds. 2006. The bacteriophages. 2nd ed. Oxford University Press, New York.

De Paepe, M., and F. Taddei. 2006. Viruses' life history: towards a mechanistic basis of a trade-off between survival and reproduction among phages. PLoS Biology 4:e193.

Dercole, F., and S. Rinaldi. 2008. Analysis of evolutionary processes: the adaptive dynamics approach and its applications. Princeton University Press, Princeton, NJ.

Edwards, K. F., and G. F. Steward. 2018. Host traits drive viral life histories across phytoplankton viruses. American Naturalist 191: $566-581$.

Ellis, E. L., and M. Delbrück. 1939. The growth of bacteriophage. Journal of General Physiology 23:365-384.

Fagerbakke, K. M., M. Heldal, and S. Norlan. 1996. Content of carbon, nitrogen, oxygen, sulfur and phosphorus in native aquatic and cultured bacteria. Aquatic Microbial Ecology 10:15-27.

Fuhrman, J. A. 1999. Marine viruses and their biogeochemical and ecological effects. Nature 399:541-548.

Geritz, S., E. Kisdi, G. Meszéna, and J. Metz. 1998. Evolutionarily singular strategies and the adaptive growth and branching of the evolutionary tree. Evolutionary Ecology 12:35-57.

Gnezda-Meijer, K., I. Mahne, M. Poljsak-Prijatelj, and D. Stopar. 2006. Host physiological status determines phage-like particle distribution in the lysate. FEMS Microbiology Ecology 55:136145.

Golec, P., J. Karczewska-Golec, M. Los, and G. Wegrzyn. 2014. Bacteriophage $\mathrm{T} 4$ can produce progeny virions in extremely slow growing Escherichia coli host: comparison of a mathematical model with the experimental data. FEMS Microbiology Letters 351:156-161.

Hadas, H., M. Einav, I. Fishov, and A. Zaritsky. 1997. Bacteriophage T4 development depends on the physiology of its host Escherichia coli. Microbiology 143:179-185.

Herendeen, S. L., R. A. VanBogelen, and F. C. Neidhardt. 1979. Levels of major proteins of Escherichia coli during growth at different temperatures. Journal of Bacteriology 139:185-194.

Husimi, Y., K. Nishigaki, Y. Kinoshita, and T. Tanaka. 1982. Cellstat: a continuous culture system of a bacteriophage for the study of the mutation rate and the selection process at the DNA level. Review of Scientific Instruments 53:517-522. 
Kokjohn, T. A., G. S. Sayler, and R. V. Miller. 1991. Attachment and replication of Pseudomonas aeruginosa bacteriophages under conditions simulating aquatic environments. Journal of General Microbiology 137:661-666.

Lennon, J. T., and J. B. H. Martiny. 2008. Rapid evolution buffers ecosystem impacts of viruses in a microbial food web. Ecology Letters 11:1178-1188.

Levin, B. R., F. M. Stewart, and L. Chao. 1977. Resource-limited growth, competition, and predation: a model and experimental studies with bacteria and bacteriophage. American Naturalist 111:3-24.

Lomas, M. W., J. Bonachela, S. A. Levin, and A. C. Martiny. 2014. Impact of ocean phytoplankton diversity on phosphate uptake. Proceedings of the National Academy of Sciences of the USA 111:17540-17545.

Maat, D. S., J. D. L. van Bleijswijk, H. J. Witte, and C. P. D. Brussaard. 2016. Virus production in phosphorus-limited Micromonas pusilla stimulated by a supply of naturally low concentrations of different phosphorus sources, far into the lytic cycle. FEMS Microbiology Ecology 92:fiw136.

Mateus, M. D. 2017. Bridging the gap between knowing and modeling viruses in marine systems - an upcoming frontier. Frontiers in Marine Science 3:284.

Menge, D. N. L., and J. S. Weitz. 2009. Dangerous nutrients: evolution of phytoplankton resource uptake subject to virus attack Journal of Theoretical Biology 257:104-115.

Middelboe, M. 2000. Bacterial growth rate and marine virus-host dynamics. Microbial Ecology 40:114-124.

Molineux, I. J. 2006. The T7 group. Pages 277-301 in R. Calendar and S. T. Abedon, eds. The bacteriophages. 2nd ed. Oxford University Press, New York.

Monod, J. 1950. La technique de culture continue, theorie et applications. Annale de l'Institut Pasteur 79:390-410.

Proctor, L. M., A. Okubo, and J. A. Fuhrman. 1993. Calibrating estimates of phage-induced mortality in marine bacteria: ultrastructural studies of marine bacteriophage development from one-step growth experiments. Microbial Ecology 25:161-182.

Rabinovitch, A., I. Fishov, H. Hadas, M. Einav, and A. Zaritsky. 2002. Bacteriophage T4 development in Escherichia coli is growth rate dependent. Journal of Theoretical Biology 216:1-4.

Rabinovitch, A., H. Hadas, M. Einav, Z. Melamed, and A. Zaritsky. 1999. Model for bacteriophage T4 development in Escherichia coli. Journal of Bacteriology 181:1677-1683.
Schulze, K. L., and R. S. Lipe. 1964. Relationship between substrate concentration, growth rate, and respiration rate of Escherichia coli in continuous culture. Archiv für Mikrobiologie 48:1-20.

Schwartz, M. 1976. The adsorption of coliphage lambda to its host: effect of variations in the surface density of receptor and in phage-receptor affinity. Journal of Molecular Biology 103:521536.

Shao, Y., and I.-N. Wang. 2008. Bacteriophage adsorption rate and optimal lysis time. Genetics 180:471-482.

Tilman, D. 1982. Resource competition and community structure. 1st ed. Princeton University Press, Princeton, NJ.

Walsh, D., and I. Mohr. 2011. Viral subversion of the host protein synthesis machinery. Nature Reviews Microbiology 9:860-875.

Wang, I.-N. 2006. Lysis timing and bacteriophage fitness. Genetics 172:17-26.

Wang, I.-N., D. E. Dykhuizen, and L. B. Slobodkin. 1996. The evolution of phage lysis timing. Evolutionary Ecology 10:545558.

Webb, V., E. Leduc, and G. B. Spiegelman. 1982. Burst size of bacteriophage sp82 as a function of growth rate of its host Bacillus subtilis. Canadian Journal of Microbiology 28:1277-1280.

Weinbauer, M. G. 2004. Ecology of prokaryotic viruses. FEMS Microbiology Reviews 28:127-181.

Weitz, J. S., and J. Dushoff. 2008. Alternative stable states in hostphage dynamics. Theoretical Ecology 1:13-19.

Weitz, J. S., H. Hartman, and S. A. Levin. 2005. Coevolutionary arms races between bacteria and bacteriophage. Proceedings of the $\mathrm{Na}$ tional Academy of Sciences of the USA 102:9535-9540.

Weld, R. J., C. Butts, and J. A. Heinemann. 2004. Models of phage growth and their applicability to phage therapy. Journal of Theoretical Biology 227:1-11.

Winter, C., T. Bouvier, M. G. Weinbauer, and T. F. Thingstad. 2010 Trade-offs between competition and defense specialists among unicellular planktonic organisms: the "killing the winner" hypothesis revisited. Microbiology and Molecular Biology Reviews 74:4257.

You, L., P. F. Suthers, and J. Yin. 2002. Effects of Escherichia coli physiology on growth of phage T7 in vivo and in silico. Journal of Bacteriology 184:1888-1894.
Associate Editor: Erol Akçay Editor: Alice A. Winn 